\title{
Norois
}

Environnement, aménagement, société

\section{Clore un conflit d'aménagement par le vote ? Note sur la géographie électorale de la consultation sur l'aéroport de Notre-Dame-des-Landes}

Can a development conflict be brought to a close by a vote? The electoral geography of the Notre-Dame-des-Landes airport consultation

\section{Jean Rivière}

\section{OpenEdition}

\section{Journals}

Édition électronique

URL : https://journals.openedition.org/norois/5921

DOI : $10.4000 /$ norois. 5921

ISBN : 978-2-7535-5227-2

ISSN : 1760-8546

\section{Éditeur}

Presses universitaires de Rennes

\section{Édition imprimée}

Date de publication : 17 octobre 2016

Pagination : 147-159

ISBN : 978-2-7535-5222-7

ISSN : 0029-182X

Référence électronique

Jean Rivière, "Clore un conflit d'aménagement par le vote ? Note sur la géographie électorale de la consultation sur l'aéroport de Notre-Dame-des-Landes », Norois [En ligne], 238-239 | 2016, mis en ligne le 17 octobre 2018, consulté le 13 janvier 2022. URL : http://journals.openedition.org/norois/5921 DOI : https://doi.org/10.4000/norois.5921 


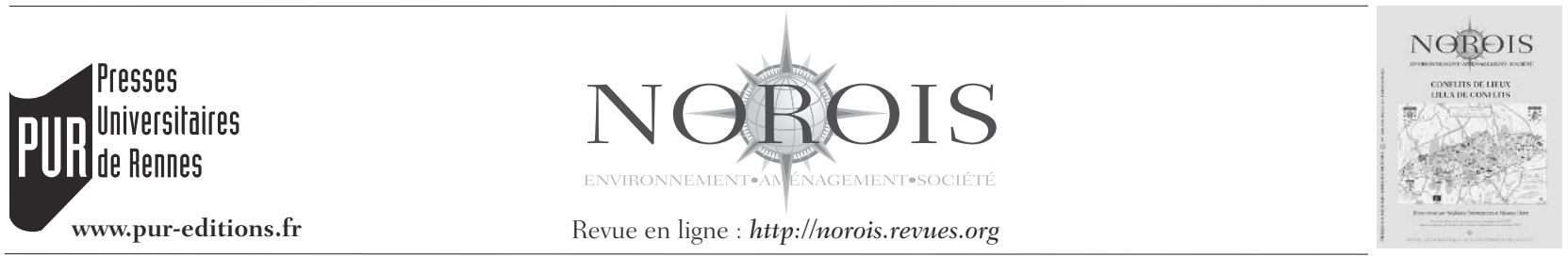

\author{
Note de recherche \\ Clore un conflit d'aménagement par le vote? \\ Note sur la géographie électorale de la consultation \\ sur l'aéroport de Notre-Dame-des-Landes \\ Can a Development Conflict be Brought to a Close by a Vote? \\ The Electoral Geography of the Notre-Dame-des-Landes Airport Consultation
}

\begin{abstract}
Jean Rivière
Maître de conférences, UMR ESO 6590 CNRS, Université de Nantes - Campus du Tertre, BP 81 227, 44312

NANTES cedex 3. (jean.riviere@univ-nantes.fr)
\end{abstract}

Résumé : La controverse politique et les mobilisations sociales autour du «transfert » de l'aéroport de Nantes-Atlantique sur la commune de Notre-Dame-des-Landes font de ce cas le plus ancien conflit d'aménagement en France. Pour tenter de clore le débat, une consultation électorale inédite a été organisée dans le département de Loire-Atlantique en juin 2016. Par un regard multi-scalaire, cette note de recherche revient sur la géographie électorale de ce scrutin lui-même controversé, en éclairant sociologiquement les résultats par des croisements statistiques avec des indicateurs du recensement de l'INSEE, et en resituant politiquement ces résultats par rapport à ceux de l'élection régionale de 2015. Il en ressort, en particulier à l'échelle intra-urbaine, que le vote « oui » renvoie plutôt aux espaces favorables aux droites tandis que le vote « non » dessine les espaces d'implantation des différentes composantes de la gauche électorale.

\begin{abstract}
The political controversy and social mobilizations surrounding the "transfer" of Nantes Atlantique airport to the village of NotreDame-des-Landes make it the longest-running development conflict in France. In an attempt to bring the debate to a close, an exceptional electoral consultation was organized across the département of Loire-Atlantique in June 2016. By adopting a multi-scalar standpoint, this research note examines the electoral geography of this controversial vote and seeks to shed light on the results in sociological terms via statistical analyses that take account of indicators from the census conducted by INSEE (the French national statistics office), and by comparing these results, in political terms, with those of the 2015 regional elections. What emerges, particularly at the intra-urban level, is that the "yes" vote generally came from spaces favorable to right-wing parties, while the "no" vote corresponded more to spaces favorable to different components of the electoral left.
\end{abstract}

Mots clés : géographie électorale - conflit - aménagement - aéroport

Keywords: electoral geography - conflict - planning - airport 


\section{UN RECOURS CONTESTÉ AU SÉSAME ÉLECTORAL}

C'est au début des années 1960 que la DATAR ${ }^{1}$ commence à envisager la création d'un aéroport international en périphérie de la ville de Nantes, qui vient alors d'être choisie comme une des « métropoles d'équilibre » visant à contrebalancer le poids parisien dans le réseau urbain français. En 1974, une ZAD (Zone d'Aménagement Différé) est créée sur le site bocager de la commune de Notre-Damedes-Landes, ce périmètre étant rebaptisé sous l'appellation militante de ZAD (Zone À Défendre) à l'automne 2012, peu après que la mise en œuvre du projet ait été accélérée par la signature d'une concession entre l'État et une filiale du groupe Vinci à la fin de l'année 2010 (Barbe, 2016). Depuis, et au fil des tentatives d'évacuation de cette ZAD par les forces de l'ordre et des recours juridiques à plusieurs échelons politico-administratifs, ce conflit est régulièrement au centre de l'agenda politicomédiatique local et national. C'est dans ce contexte de contestation sociale multiforme qu'a finalement été annoncée, au printemps 2016, la tenue d'une consultation électorale locale - qui n'a pas valeur de référendum - dans le périmètre du département de Loire-Atlantique, cette annonce ouvrant elle-même de nouveaux litiges.

\section{Un espace découpé sur mesure}

La légitimité politique du choix de ce périmètre, défendu par le Premier ministre Manuel Valls, a en effet été discutée à la fois pas plusieurs associations d'opposants au projet, mais aussi par la ministre de l'Environnement Ségolène Royal (qui a proposé de l'élargir aux départements limitrophes) et par les présidents des conseils départementaux voisins réclamant que leurs habitants soient consultés sur un projet qu'ils contribuent à financer ${ }^{2}$. Pour les géographes qui interrogent les échelles des pouvoirs, cette contestation multi-scalaire est d'autant plus intéressante que plusieurs articles de presse parus avant et après la consultation ont souligné combien

1. Délégation à l'Aménagement du Territoire et à l'Action Régionale.

2. Le site [www.nddl-debatpublic.fr] visant à informer les électeurs rappelle en effet que sur les 115 millions d'euros prévus au titre de l'engagement des collectivités territoriales pour financer ce projet, $25 \%$ sont apportés par le Conseil régional de Bretagne (dont les habitants ne sont pas invités à donner leur avis) et $35 \%$ par celui des Pays de la Loire (dont les habitants de quatre départements sur cinq ne sont pas appelés à se prononcer). le choix du périmètre du département pouvait traduire des arrière-pensées électoralistes. En effet, et sur la base de sondages d'intention de vote dont la non-validité scientifique est certes largement établie en sociologie critique (Garrigou, 2006; Lehingue, 2007) - mais que l'on peut tout de même comparer grossièrement pour les sondages réalisés par le même institut -, ces articles de presse soulignent que le département est l'espace de consultation le plus favorable au «oui » parmi les coups de sonde réalisés selon différents périmètres ${ }^{3}$. À seulement six jours du scrutin, le Conseil d'État a finalement jugé qu'il était juridiquement valable que l'aire couverte par la consultation du 26 juin 2016, soit celle du département dans la mesure où la préfecture de Nantes avait été désignée comme lieu de l'enquête publique en 2006, soit dix ans plus tôt.

\section{Des modalités de " campagne » en question}

Par ailleurs, la manière dont a été organisé (ou plutôt n'a pas été organisé) le « débat public » visant à informer équitablement l'ensemble des citoyens appelés à s'exprimer lors de cette consultation, a également fait l'objet de contestations relayées par la presse ${ }^{4}$, en particulier sur les conditions d'encadrement de la «campagne » ou sur l'absence de documents papiers officiels envoyés aux électeurs (le courrier de la Préfecture appelant à participer à la consultation renvoyait les électeurs exclusivement vers un site Internet ${ }^{5}$, ce qui fait jouer à plein l'inégale maîtrise des outils numériques en fonction des groupes sociaux). Les mots n'étant pas neutres dans la manière de poser les termes d'un débat politique, c'est jusqu'à la question soumise aux électeurs ( Êtes-vous favorable au projet de transfert de l'aéroport de Nantes-Atlantique sur la commune de Notre-Dame-des-Landes? ») qui a fait l'objet de recours d'associations d'opposants et d'habitants devant le Conseil d'État, et en particulier l'usage du terme de «transfert » dans la mesure où l'actuel aéroport de Nantes-Atlantique continuera à être utilisé - à raison de quelques vols hebdomadaires -

\footnotetext{
3. Voir par exemple : «Référendum sur Notre-Dame-des-Landes : et si le gouvernement avait choisi une autre échelle? ", Marianne, 28 juin 2016.

4. « Notre-Dame-des-Landes : une campagne d'information à armes inégales », Mediapart, 7 juin 2016.

5. « Notre-Dame-des-Landes : les drôles de lacunes du site de la campagne », Mediapart, 11 juin 2016.
} 
dans le cadre des activités industrielles du groupe Airbus. Enfin, la question posée évoque également un " projet », sans qu'il ne soit explicitement précisé quelle version du projet est soumise au vote (une ou deux pistes). Sur ces deux points de litige, le Conseil d'État a également tranché en considérant qu'il était clair « que le projet soumis à consultation est le projet qui avait été déclaré d'utilité publique en 2008 », donc celui à deux pistes pourtant jugé « surdimensionné » par les experts du ministère de l'Environnement en avril $2016^{6}$, et que plus largement la «sincérité du scrutin à venir » ne pouvait être mise en cause.

Dans l'ensemble, et comme l'a relevé un politiste, c'est toutefois une impression de «bricolage juridique permanent » (Boudic, 2016) qui se dégage des dernières péripéties autour de ce projet d'aménagement controversé. Loin d'être anecdotiques tant on en mesure les effets sur l'issue du scrutin, ces questions devaient être rappelées avant d'esquisser la socio-géographie électorale de cette consultation inédite.

\section{Du silence des géographes... à leur (tardive) expression publique}

Mais avant cela, un dernier détour se doit d'être proposé au lecteur. En effet, et si l'histoire du conflit d'aménagement lié à la construction du nouvel aéroport de Nantes sur la commune de Notre-Damedes-Landes commence désormais à être bien documentée, les sciences sociales y ont surtout brillé - jusqu'à récemment (Renard et Rialland-Juin, 2013; Pailloux, 2015) - par leur absence (Barbe, 2016). Cette absence des sciences sociales ne peut que questionner les postures des enseignants-chercheurs, en particulier celles des géographes qui ont plutôt endossé le rôle de " conseillers du prince » dans l'histoire française de l'aménagement, au point que cette collusion ancienne soit dénoncée au milieu des années 1970 dans le cadre du mouvement de renouvellement de la géographie universitaire : "Nous accusons la géographie dominante d'être complice de l'ordre social/spatial établi, quand elle le légitime ou quand elle l'aménage. Nous lui reprochons autant ses discours que ses silences ». (Collectif Hérodote, 1976). Face à un tel objet de

6. « Notre-Dame-des-Landes : le projet d'aéroport est "surdimensionné" selon les experts », Le Monde, 5 avril 2016. recherche, il semble donc nécessaire de préciser d'où l'on parle (encadré).

\section{Une démarche méthodologique classique en géographie électorale}

Cette note de recherche s'attache à proposer des éléments de compréhension des résultats de cette consultation en mobilisant des matériaux empiriques agrégés selon deux échelles d'analyse : celle des 212 communes du département de LoireAtlantique (le périmètre d'organisation du scrutin étant donc couvert exhaustivement) et celle des 200 bureaux de vote intra-urbains de la ville de Nantes ${ }^{7}$. Son objectif consiste, dans un premier temps, à dresser une cartographie académique des résultats du scrutin, pour dépasser les premières images publiées rapidement dans les médias régionaux et nationaux, et dont la construction et la sémiologie posent un certain nombre de problèmes qui sont tout sauf politiquement neutres : cartes choroplètes qui ne restituent pas - à l'exception de celles proposées par Mediapart ${ }^{8}$ - l'inégal poids démographique des communes; cartes qui ne proposent parfois que deux classes distinguant seulement les communes où le « oui » et le «non » sont majoritaires, sans proposer de classes nuançant l'intensité des scores; cartes qui reposent presque exclusivement sur des pourcentages de « oui » et de «non » établis sur les électeurs inscrits, alors que les $51 \%$ de participation invitent justement à tenir compte du poids de l'abstention.

Dans un second temps, il s'agira de proposer quelques éléments de sociologie électorale de ce scrutin, en établissant pour cela - de manière classique en géographie - des corrélations statistiques entre les comportements électoraux relevés lors de la consultation (abstention, votes « oui »

7. Il aurait été utile de pouvoir utiliser un fond de carte de l'ensemble des bureaux de vote de Nantes-Métropole, notamment pour pouvoir analyser à l'échelle intra-communale les résultats dans les villes de Rezé et de Bouguenais (les plus exposées aux nuisances de l'aéroport de NantesAtlantique), mais le fait que le redécoupage des bureaux de vote relève du domaine de compétences de chaque commune implique des contraintes importantes de mise à jour de tels fonds de cartes métropolitains (Bussi et al., 2010).

8. « Notre-Dame-des-Landes : un Oui teinté de Non », Mediapart, 27 juin 2016. En raison du travail d'investigation mené par ce quotidien depuis son lancement et de sa porosité aux objets des sciences sociales, j'ai contacté leur rédaction pour leur proposer une analyse « à chaud » des résultats de la consultation (voir : "Notre-Dame-des-Landes : un non de gauche », Médiapart, 29 juin 2016) parce qu'il me semblait que c'était important en matière d'utilité sociale de la recherche académique. 
Mes travaux s'inscrivent dans le cadre d'une géographie sociale centrée sur l'analyse de la dimension spatiale des inégalités sociales et des rapports de pouvoir et de domination. Ma thèse a notamment été encadrée par Robert Hérin, qui a contribué à l'installation de ce courant dans le champ de la géographie française et qui l'a toujours situé au regard d'une posture «impliquée et militante », en particulier autour de la nécessité de dévoiler les logiques de reproduction des inégalités dans l'espace pour les dénoncer et les combattrea.

Suite à mon recrutement à Nantes en 2011 , j’ai été frappé - tout en étant conscient que l'histoire de la discipline apportait des clés pour le comprendre - par le fait qu'aucun collègue en activité ne travaille sur le conflit de NotreDame-des-Landes, qui me semblait pourtant constituer un terrain d'enquête idéal pour réunir les différentes sensibilités des géographes. Depuis, j’ai pourtant croisé nombre de ces collègues lors des manifestations locales contre le projet d'aéroport et je crois, en fait, n'avoir jamais échangé qu'avec des géographes nantais opposés à ce projet, avec des arguments certes différents. Pour ne pas regretter de ne pas avoir essayé (et non sans un certain goût pour la provocation), j'ai envoyé - une dizaine de jours avant la consultation de juin 2016 - un courriel à l'ensemble des collègues de l'IGARUN $N^{\mathrm{b}}$ pour initier la rédaction d'une tribune de presse collective appelant à voter « non » à ce scrutin, bien que les modalités de son organisation le rendent à mes yeux illégitime. Il faudrait interroger plus en détail que dans ce rapide encadré la manière dont une telle proposition bouscule les positionnements épistémologiques et politiques des uns et des autres, mais il me semble que pour les collèguesc ${ }^{c}$, cette proposition a suscité un certain enthousiasme et a - ponctuellement et modestement - contribué à redonner un peu de sens à une activité professionnelle profondément affectée par la bureaucratisation de l'institution universitaire.

Enfin et si l'attitude qui consiste à dire ce qu'un chercheur vote avant de produire une analyse scientifique du vote des autres me semble saine intellectuellement, elle n'en reste pas moins rare dans le petit monde du commentaire électoral (et c'est d'ailleurs la première fois que je me livre moi-même à cet exercice réflexif).

a. C'est dans cet esprit que les principaux résultats empiriques de cette note de recherche ont été présentés aux acteurs et militants mobilisés contre le projet d'aéroport lors du rassemblement annuel «Semailles de démocratie » ayant eu lieu sur la ZAD le week-end du 9-10 juillet 2016.

b. Institut de Géographie et d'Aménagement Régional de l'Université de Nantes.

c. Notre tribune a été signée par une large majorité des collègues et finalement publiée dans le principal quotidien régional à trois jours du scrutin «NDDL. L'appel à voter "non" dimanche d'un collectif de géographes », Ouest-France, 23 juin 2016.

Se situer politiquement face à un objet politique

Political positions in the face of a political object

et vote «non » $)^{9}$ et des indicateurs sociaux issus du recensement de la population de l'INSEE de 2012 (tranches d’âge, niveaux de diplômes, catégories socioprofessionnelles, statut d'occupation des logements $)^{10}$. Rappelons deux choses. D'une part, que les deux types de données mises en relation ne recouvrent pas exactement les mêmes champs de populations puisque les résultats électoraux portent sur les seuls inscrits, tandis que les données du recensement de l'INSEE décrivent la structure

9. Une cartographie des bulletins blancs ou nuls, désormais décomptés séparément, a été établie ainsi que des corrélations calculées de manière exploratoire. Ces résultats qui n'étaient pas significatifs ne sont pas présentés dans cette note. Une analyse des votes par procurations serait par contre intéressante à conduire sur ce type de scrutin, notamment à l'échelle intra-urbaine, et ce dans l'esprit de premiers travaux en cours sur la question (Charpentier, Coulmont, Gombin, 2014).

10. L'analyse à l'échelle communale ne pose pas de problèmes méthodologiques spécifiques, contrairement à celle menée à l'échelle intra-urbaine dans Nantes. En effet et dans la mesure où les résultats électoraux sont collectés à l'échelle des bureaux de vote et que les indicateurs sociologiques issus du recensement de l'INSEE sont disponibles à l'échelle des IRIS, une procédure de géomatique développée dans le cadre de l'ANR CARTELEC a permis de ventiler les données depuis la maille spatiale des IRIS vers la trame géographique des bureaux de vote, ce afin de pouvoir établir des corrélations statistiques (Beauguitte, Colange, 2013). de l'ensemble de la population résidente (dont les étrangers, les Français privés de leurs droits civiques et les Français non-inscrits). D'autre part, le fait de travailler au niveau des communes ou des bureaux de vote ne fait pas disparaître le fameux risque «d'erreur écologique » (Robinson, 1950): on sait en effet depuis longtemps que les analyses qui croisent la composition sociologique des espaces avec les choix électoraux de ceux qui y vivent ne peuvent pas prouver qu'il existe un lien entre votes et propriétés sociales au niveau des individus. Ce risque diminue toutefois quand on raisonne à l'échelle la plus fine, celle du bureau de vote.

Enfin, le troisième objectif consiste, avec les mêmes techniques statistiques et les mêmes enjeux méthodologiques, à tenter d'éclairer politiquement les résultats de cette consultation en les croisant avec ceux du scrutin régional de $2015^{11}$. C'est en

\footnotetext{
11. Sur le plan méthodologique, c'est aussi le seul scrutin qu'il est possible de croiser statistiquement avec la consultation, car les bureaux de vote de la ville de Nantes ont été redécoupés en janvier 2015.
} 
effet le scrutin le plus proche dans le temps de la consultation de juin 2016, et cela semble d'autant plus pertinent que la dernière élection régionale dans la région Pays de la Loire a été largement marquée par l'enjeu du projet d'aéroport ${ }^{12}$. Ce sont notamment les rapports entre les différentes composantes de la gauche électorale qui ont été les plus affectés par cet enjeu : il a constitué une source de désaccord majeur entre la liste conduite par le PS (favorable au projet) et celle d'EELV (opposée au projet), ces deux listes fusionnant tout de même entre les deux tours ${ }^{13}$; et il a participé à l'éclatement local de la liste qui devait être présentée par le Front de gauche, le PCF de Loire-Atlantique soutenant le projet, considéré comme inacceptable par le PG et Ensemble - deux des autres principales composantes de cette coalition électorale -, le PCF conduisant finalement la liste avec le soutien du MRC.

Le plan de cette note de recherche est construit selon une logique thématique, et s'intéresse successivement à la question de l'abstention, du vote « oui » et du vote «non », en mobilisant à chaque fois les deux échelles d'analyse (communale et intraurbaine).

\section{UN SCRUTIN BANAL EN MATIÈRE D'ABSTENTION}

Cette consultation électorale, inédite sur un grand projet d'aménagement, a finalement donné lieu à une abstention de près de la moitié (49\%) des électeurs inscrits. Le travail d'exégèse savante et politique de cette donnée est bien entendu au cœur des luttes d'interprétation du résultat (Lehingue, 2005). D'un côté, le Premier ministre Manuel Valls a salué « le taux exceptionnel de participation de $51 \%$, validant l'intérêt de cette consultation ${ }^{14}{ }^{»}$. De l'autre, la coordination des opposants au projet peut s'appuyer

12. Voir par exemple : « Batailles autour d'un aéroport », supplément « Régionales 2015 - Pays de la Loire », Le Monde, 23 octobre 2015.

13. Le texte de l'accord électoral entre les deux listes comprend ainsi une annexe spécifique au projet qui commence par la phrase suivante : « Le projet de Notre-Dame-des-Landes fait l'objet d'un fort désaccord entre écologistes et socialistes depuis de nombreuses années. La liste conduite par Christophe Clergeau réaffirme son soutien à la réalisation de ce projet; celle conduite par Sophie Bringuy, son opposition à ce projet, et son soutien à l'optimisation de Nantes-Atlantique ». Voir : « Régionales. Accord pour une fusion PS-EELV en Pays de la Loire », Ouest-France, 7 décembre 2015.

14. « Notre-Dame-des-Landes : Manuel Valls prévient les occupants illégaux qu'ils "devront partir" ", Le Monde, 26 juin 2016. sur le fait qu'un inscrit sur deux n'a pas voté pour continuer de contester la légitimité du projet comme de la consultation elle-même.

Avec un regard académique, on peut considérer que cette consultation constitue un scrutin dit de faible intensité qui le place dans la continuité de nombre de scrutins locaux des années 2000, caractérisés par une abstention qui tend à devenir le nouveau «vote normal» - au sens de norme statistique ou de comportement modal - pour reprendre une expression du politiste Patrick Lehingue. À titre de comparaison, le scrutin départemental de mars 2015 a été marqué par une abstention de $51 \%$ en Loire-Atlantique, tandis que le scrutin régional de décembre 2015 a vu une abstention assez proche (42\%) dans le même département. Les cartographies de ce taux d'abstention mettent en évidence des clivages géographiques profonds, et ce aux deux échelles d'analyse considérées (figure 1 - planche IX).

\section{Un département profondément divisé en matière de participation}

Au niveau départemental, il en ressort que la proximité à la commune de Notre-Dame-desLandes a favorisé la participation à la consultation, ce qui n'est pas une surprise au regard des enjeux pour partie très localisés que soulève un tel projet (nuisances de toutes sortes, restructuration parcellaire, modification des infrastructures, du cadre paysager...). À l'inverse, l'abstention a été particulièrement forte au niveau de l'estuaire de la Loire et de l'agglomération urbaine populaire de Saint-Nazaire, le tableau 1 soulignant un coefficient de corrélation positif avec la part communale de locataires du parc locatif HLM, qui est un indicateur classiquement utilisé pour saisir la précarité sociale, dont il est établi qu'elle éloigne durablement de l'institution électorale (Braconnier, Mayer, 2015). Plus généralement, l'abstention a été élevée au sein d'une large bande littorale du département, qui compte de nombreuses résidences secondaires (d'où un phénomène probable de mal-inscription sur les listes électorales accentuant l'abstention) et une proportion importante de retraités (les coefficients de corrélation $r$ sont légèrement positifs entre l'abstention et la présence des 65-79 ans et des plus de 80 ans, et de la $\mathrm{CSP}$ « retraités»). 


\begin{tabular}{|c|c|c|}
\hline Variables & $\begin{array}{l}\text { Échelle des } \\
\text { communes }\end{array}$ & $\begin{array}{c}\text { Échelle des } \\
\text { bureaux }\end{array}$ \\
\hline 18-24 ans & NS & NS \\
\hline 25-39 ans & $-0,21$ & NS \\
\hline $40-54$ ans & NS & 0,18 \\
\hline 55-64 ans & NS & NS \\
\hline 65-79 ans & 0,20 & NS \\
\hline 80 ans ou plus & 0,17 & $-0,16$ \\
\hline Sans diplôme & NS & 0,54 \\
\hline CEP & NS & 0,24 \\
\hline BEPC & 0,16 & 0,23 \\
\hline CAP-BEP & NS & 0,28 \\
\hline $\mathrm{Bac}$ & NS & $-0,15$ \\
\hline $\mathrm{Bac}+2$ & NS & $-0,56$ \\
\hline Bac +3 et plus & NS & $-0,34$ \\
\hline Agriculteurs & $-0,28$ & 1 \\
\hline $\begin{array}{l}\text { Artisans, comm., chefs } \\
\text { entr. }\end{array}$ & NS & NS \\
\hline Cadres, Prof. intel. sup. & NS & $-0,43$ \\
\hline Prof. Intermédiaires & NS & $-0,51$ \\
\hline Employés & NS & 0,36 \\
\hline Ouvriers & NS & 0,48 \\
\hline Retraités & 0,19 & NS \\
\hline Autres & NS & NS \\
\hline Propriétaires & $-0,28$ & $-0,53$ \\
\hline Locataires parc privé & NS & $-0,28$ \\
\hline Locataires parc HLM & 0,31 & 0,55 \\
\hline Logés gratuitement & NS & NS \\
\hline Abstention & 0,29 & 0,93 \\
\hline Blancs & $-0,16$ & NS \\
\hline Nuls & NS & NS \\
\hline $\mathrm{LO}$ & NS & 0,20 \\
\hline $\mathrm{CO}$ & 0,15 & 0,21 \\
\hline PCF-MRC & NS & NS \\
\hline EELV & $-0,52$ & $-0,53$ \\
\hline PS-PRG & NS & $-0,56$ \\
\hline UDB & $-0,15$ & $-0,30$ \\
\hline UMP-UDI-MODEM & NS & $-0,58$ \\
\hline UPR & NS & $-0,22$ \\
\hline DLF & NS & $-0,44$ \\
\hline FN & $-0,19$ & NS \\
\hline
\end{tabular}

Tableau 1 : Coefficients de corrélation a entre l'abstention et des variables sociales et politiques à l'échelle communale et des bureaux de vote nantais

Ecological correlation between abstention and political sociological variables by municipality and by polling station in Nantes
Secondairement, l'abstention a été assez importante au cœur de Nantes-Métropole, notamment à Nantes, Saint-Herblain ou Rezé, qui sont certes des communes très peuplées dans lesquelles l'abstention est souvent élevée quel que soit le type de scrutin (le $\mathrm{r}$ entre l'abstention à la consultation et l'abstention au $1^{\text {er }}$ tour des régionales 2015 est de $0,29)$, mais qui constituent aussi le cœur métropolitain auquel est destiné le projet d'aéroport. Le projet n'y a donc pas suscité la mobilisation électorale massive à laquelle on aurait pu s'attendre. De manière plus générale, les corrélations statistiques établies au niveau communal sont globalement assez faibles par rapport à l'échelle fine des bureaux de vote (Russo, Beauguitte, 2012).

\section{Dans l'espace nantais, des déterminants sociaux qui pèsent à plein}

La carte de l'abstention à l'échelle des bureaux de vote nantais (figure 2) est en effet bien connue puisqu'elle correspond à celle que l'on pourrait dresser à l'issue de chaque scrutin, comme l'illustre la corrélation spectaculaire de 0,93 entre l'abstention lors de la consultation et celle lors des régionales 2015. Cette carte renvoie à la localisation des principaux quartiers populaires de grands ensembles dans l'espace urbain. Ainsi, dans certains bureaux des Dervallières, de Bellevue, de Nantes-Nord ou de Malakoff, l'abstention se situe fréquemment entre 70 et $80 \%$ des inscrits (Braconnier, Dormagen, 2007). Les corrélations établies avec les indicateurs de l'INSEE montrent clairement les relations statistiques entre l'abstention intra-urbaine et la présence des populations les moins bien situées dans les hié-

a. Les valeurs du coefficient $r$ varient toujours entre - 1 et 1 . La première information est donnée par le signe de la corrélation. Lorsque $\mathrm{r}$ est positif, le niveau d'abstention a tendance à s'élever quand la proportion d'une catégorie dans la population (par exemple les « 18 24 ans ») augmente. A l'inverse quand r est négatif, le pourcentage d'abstention diminue quand la part de cette catégorie dans la population augmente. La seconde information est fournie par la valeur du coefficient r. Quand cette valeur tend vers -1 ou 1, cela signifie que la relation statistique entre l'abstention et la catégorie sociale dont on teste les effets est très intense. Par contre et quand la valeur de $r$ est proche de 0 , on peut en conclure qu'il n'existe pas de relation entre l'abstention et cette catégorie sociale. Enfin, on peut tester la significativité de la corrélation établie afin d'en évaluer la robustesse statistique. Toutes les corrélations présentées dans les tableaux 1, 2 et 3 sont significatives avec une marge d'erreur inférieure à $5 \%$, celles significatives avec une marge d'erreur de $0,1 \%$ sont signalées en gras. Quand ce n'est pas le cas, une mention NS (pour « nonsignificatif ») le précise. 
rarchies scolaires ( $\mathrm{r}=0,54$ avec les sans-diplômes), professionnelles $(0,48$ avec les ouvriers et 0,36 avec les employés) et résidentielles $(0,55$ avec les locataires du parc HLM). En cela, cette consultation constitue bien sociologiquement un scrutin banal.

\section{LE VOTE « OUI » : DES MARGES RURALES AUX BEAUX QUARTIERS NANTAIS}

Comme espéré par le gouvernement une fois le périmètre de la consultation circonscrit au cadre départemental, c'est finalement le « oui » qui l'a emporté par $55 \%$ des suffrages exprimés, soit un gros quart (28\%) des électeurs inscrits. Si le Premier Ministre s'est réjouit de ce résultat en considérant que « la démocratie a parlé ${ }^{15}$ », les opposants au projet pourront lui objecter que la majorité $(72 \%)$ des inscrits n'ont pas voté « oui » lors de cette consultation. Symétriquement, et dans la mesure où seuls $23 \%$ des inscrits ont voté «non », il est possible d'affirmer que plus des trois-quarts $(77 \%)$ des inscrits n'ont pas refusé ce projet dans les urnes. Dès lors, on comprend qu'il ne s'agit pas tant de mettre en cause la légalité du résultat final établi sur la base des suffrages exprimés - qui sert généralement de base pour répondre à la question politique et médiatique du «qui a gagné » (Lehingue, 2005) - que de porter un regard de sciences sociales prenant pleinement en compte les effets des dynamiques d'abstention sur la socio-géographie des résultats. L'analyse des géographies du vote « oui » est en cela intéressante (figure 2 - planche $\mathbf{X}$ ).

\section{Un vote " oui » concentré dans les péri- phéries rurales du nord du département}

La géographie du vote oui est nettement structurée par un gradient opposant les marges nord du département au reste de la Loire-Atlantique ${ }^{16}$,

15. « Notre-Dame-des-Landes : Manuel Valls prévient les occupants illégaux qu'ils «devront partir» », Le Monde, 26 juin 2016.

16. Le contraste est si net qu'une pétition (ayant recueilli près de 10000 signatures au 15 juillet 2016) intitulée « Pour le déplacement de l'aéroport de NDDL " a été mise en ligne, elle s'appuie de manière humoristique sur une carte des résultats qui localise un autre site géographique possible pour l'aéroport au cœur de l'espace le plus favorable au « oui ». La carte comprend une flèche pointant vers ce site imaginaire et mentionne : «Afin de satisfaire les pour et les contre, l'aéroport sera déplacé dans cette zone où tout le monde semble d'accord pour qu'il se fasse », voir : [https://www.change.org/p/pour-le-d\%C3\%A9placement-del-a\%C3\%A9roport-de-nddl].

\begin{tabular}{|c|c|c|}
\hline Variables & $\begin{array}{l}\text { Échelle des } \\
\text { communes }\end{array}$ & $\begin{array}{c}\text { Échelle des } \\
\text { bureaux }\end{array}$ \\
\hline $18-24$ ans & NS & NS \\
\hline $25-39$ ans & NS & $-0,34$ \\
\hline $40-54$ ans & $-0,16$ & NS \\
\hline $55-64$ ans & NS & 0,28 \\
\hline $65-79$ ans & NS & 0,20 \\
\hline 80 ans ou plus & NS & 0,14 \\
\hline Sans diplôme & 0,37 & $-0,33$ \\
\hline CEP & 0,41 & NS \\
\hline $\mathrm{BEPC}$ & NS & NS \\
\hline CAP-BEP & NS & NS \\
\hline $\mathrm{Bac}$ & $-0,28$ & NS \\
\hline $\mathrm{Bac}+2$ & $-0,39$ & 0,30 \\
\hline Bac +3 et plus & $-0,36$ & 0,17 \\
\hline Agriculteurs & 0,50 & 1 \\
\hline $\begin{array}{l}\text { Artisans, comm., chefs } \\
\text { entr. }\end{array}$ & NS & NS \\
\hline Cadres, Prof. intel. sup. & $-0,33$ & 0,28 \\
\hline Prof. Intermédiaires & $-0,37$ & 0,26 \\
\hline Employés & NS & $-0,24$ \\
\hline Ouvriers & 0,33 & $-0,34$ \\
\hline Retraités & NS & 0,20 \\
\hline Autres & NS & NS \\
\hline Propriétaires & NS & 0,57 \\
\hline Locataires parc privé & NS & NS \\
\hline Locataires parc HLM & $-0,21$ & $-0,33$ \\
\hline Logés gratuitement & NS & NS \\
\hline Abstention & $-0,14$ & $-0,77$ \\
\hline Blancs & 0,23 & NS \\
\hline Nuls & 0,36 & NS \\
\hline LO & 0,15 & $-0,27$ \\
\hline $\mathrm{CO}$ & $-0,17$ & $-0,20$ \\
\hline PCF-MRC & $-0,24$ & $-0,24$ \\
\hline EELV & $-0,23$ & NS \\
\hline PS-PRG & $-0,21$ & 0,28 \\
\hline UDB & NS & NS \\
\hline UMP-UDI-MODEM & NS & 0,68 \\
\hline UPR & NS & 0,17 \\
\hline DLF & NS & 0,51 \\
\hline FN & 0,38 & 0,33 \\
\hline
\end{tabular}

Tableau 2 : Coefficients de corrélation entre le vote « oui » et des variables sociales et politiques à l'échelle communale et des bureaux de vote nantais

Ecological correlation between the "yes » vote and political/ sociological variables by municipality and by polling station in Nantes 
même si le « oui » dépasse le seuil de $50 \%$ des inscrits dans seulement quatre communes qui comptent chacune quelques centaines d'habitants. Une hypothèse explicative qui pourrait être avancée pour comprendre ce gradient tient dans le fait que plusieurs élus locaux de la région de Chateaubriand ont soutenu le projet d'aéroport, l'un de leurs arguments reposant sur la construction d'une liaison en tram-train qui pourrait être connectée à l'actuelle ligne Nantes-Chateaubriand, proposant une hypothétique desserte en transport en commun vers le site de Notre-Dame-des-Landes. Le coût de cette liaison (estimé entre 150 et 200 millions d'euros) n'est toutefois pas intégré au projet d'aéroport (luimême chiffré à 550 millions d'euros) tel que soumis à la consultation, et aucune décision ni calendrier n'ont été arrêtés sur cet enjeu pourtant majeur.

Un autre élément qui ressort de la figure 2 (planche $\mathbf{X}$ ) réside dans l'absence de soutien massif au projet de "transfert » de l'aéroport au niveau des communes riveraines de l'aéroport actuel. On aurait en effet pu faire l'hypothèse que les habitants des espaces les plus affectés par les nuisances, notamment sonores, de l'aéroport de Nantes-Atlantique optent massivement pour le « oui ». Or, il n'en est rien. Hormis à SaintAignan-de-Granlieu où le « oui » obtient $72 \%$ des exprimés ( $47 \%$ des inscrits) et dans quelques communes de la deuxième couronne de NantesMétropole (comme Bouaye, Saint-Léger-lesVignes, Pont-Saint-Martin, Les Sorinières où le « oui » oscille entre 30 et $37 \%$ des inscrits), les résultats indiquent que l'adhésion au « transfert » de l'aéroport y est moins forte qu'attendue.

Les corrélations établies à l'échelle communale (tableau 2) - qui sont à manier avec beaucoup de précaution ${ }^{17}$ - soulignent des liens statistiques entre le vote " oui » et la présence des groupes sociaux populaires ( $r=0,33$ avec les ouvriers), ainsi qu'avec

17. Un bon exemple de risque de surinterprétation réside dans la corrélation forte et positive $(0,50)$ entre la présence de la CSP des agriculteurs et le vote «oui ", qui pousserait le chercheur pressé à en conclure que les agriculteurs ont plus voté oui que d'autres CSP. En effet il est assez contre-intuitif de penser que les agriculteurs, qui constituent a priori un groupe - certes hétérogène sociologiquement - peu favorable à la consommation de foncier agricole pour l'urbanisation aurait massivement penché pour le projet d'aéroport. Ce qu'une telle corrélation permet en fait de dire, c'est seulement que plus les agriculteurs sont présents dans une commune, plus le vote « oui » y est fort; or, les agriculteurs sont simplement plus concentrés dans les communes rurales (où l'on vient de montrer que le « oui » est élevé) mais rien n'indique que ce sont eux qui y ont voté « oui ». celle les populations faiblement diplômées $(0,37$ avec les non-diplômés et 0,41 avec les titulaires du certificat d'études primaires, ce qui renvoie en fait à un effet de génération lié à la présence de populations âgées dans ces communes). Politiquement, le « oui » est d'autant plus fort que les habitants des communes avaient opté pour des votes blancs $(\mathrm{r}=0,23)$ ou nuls $(0,36)$ et pour le FN $(0,38)$ lors du scrutin régional de 2015. Ce point est intéressant par rapport à la position originale du FN, qui avait appelé à la fois à voter «non » à ce projet tout en appelant à l'évacuation des «zadistes », le terme étant plus que péjoratif dans le discours de cette formation politique ${ }^{18}$. On peut alors poser l'hypothèse, qui appelle bien sûr confirmation par des travaux qualitatifs localisés, que dans ces mondes ruraux populaires éloignés de la métropole nantaise, le vote « oui » pourrait traduire en partie cette volonté d'expulsion de la ZAD. S'ils doivent également être interprétés avec prudence, les éléments empiriques livrés par l'analyse intra-urbaine du vote « oui » sont cependant plus clairs.

\section{Un vote « oui » qui dessine l'espace nantais des droites}

Comme pour la carte de l'abstention, celle du vote « oui » dans les quartiers nantais est bien connue des observateurs de la vie politique locale (figure 2 - planche $\mathbf{X}$ ) tant elle correspond aux bastions des différentes composantes des droites. Le centre-ouest de la ville-centre (quartiers de Procé, de HautsPavés, de Saint-Félix) est l'espace des quartiers de la droite traditionnelle (Rapetti, 1985), tandis que le quart nord-est (depuis Saint-Donatien jusqu'à la partie nord du quartier Nantes-Erdre) renvoie à la fois à des espaces de la droite classique mais aussi à des zones d'implantation plus récente du FN. Les corrélations saisies au niveau des bureaux de vote confirment largement cette impression visuelle, puisque le vote « oui » à la consultation est très lié statistiquement $(r=0,68)$ aux suffrages accordés à la liste conduite aux régionales 2015 par Bruno

18. La tête de liste du FN aux régionales 2015 avait en effet déclaré : « Notre opposition à ce projet fou est résolue. Notre-Dame-des-Landes est une aberration et même un danger pour l'avenir de notre région. Les zadistes? La violence et la terreur imposées par ces extrémistes marginaux de l'ultragauche sont inadmissibles. Ils doivent être expulsés ", voir « Notre-Dame-des-Landes. Le FN ne veut ni aéroport ni zadistes », Ouest-France, 20 octobre 2015. 
Retailleau (LR-UDI-MODEM), l'actuel Président de Région et président du groupe parlementaire LR au Sénat, qui exhorte l'État à expulser la ZAD depuis sa prise de mandat. Le vote « oui » apparaît également lié aux espaces où la liste de Debout la France (DLF) avait recueilli des scores élevés $(\mathrm{r}=0,51)$, ainsi qu'à ceux plutôt favorables au FN $(0,33)$, ce qui confirme le résultat obtenu avec les corrélations communales. Dans le même temps, ce vote « oui » est légèrement relié $(\mathrm{r}=0,28)$ aux résultats de la liste de Christophe Clergeau (PS-PRG), ce qui n'est pas étonnant tant l'ensemble de l'appareil politique du PS n'aura pas ménagé sa peine pour promouvoir le projet d'aéroport... même si la dernière partie de cette note relativise la portée de cette liaison statistique.

Sociologiquement, les corrélations établies dressent le portrait des quartiers les plus favorables aux droites dans l'espace intra-urbain. Le vote « oui » y est ainsi corrélé avec la présence d'habitants âgés ( $r=0,28$ avec les 55-64 ans et 0,20 avec les 65-79 ans), des propriétaires de leur logement $(0,57)$, et des CSP des classes moyennes et supérieures $(0,28$ avec les cadres et professions intellectuelles supérieures et 0,26 avec les professions intermédiaires), même si ces deux dernières corrélations n'atteignent pas des niveaux très élevés, ce sur quoi on reviendra.

\section{LE VOTE « NON » : UN EFFET DE PROXIMITÉ... MAIS PAS SEULEMENT}

Face à une consultation électorale tardivement imposée par l'État, qu'elle a d'emblée rejetée et dont elle avait annoncé à l'avance qu'elle considérait son résultat comme un avis parmi d'autres dans ce dossier d'aménagement, la coordination des opposants au projet a finalement décidé de faire campagne pour le «non » (plutôt que d'appeler au boycott par exemple) pour « profiter de cette tribune pour informer », afin que «la population puisse voter en toute connaissance de cause ${ }^{19} »$. Finalement, le « non » n'est majoritaire parmi les suffrages exprimés que dans une trentaine de commune, et il dépasse la barre des $50 \%$ des inscrits dans deux communes seulement : Notre-Dame-des-Landes

19. Communiqué de presse du 22 avril 2016 intitulé « Position de l’ACIPA sur la "consultation" », en ligne sur : [https:/hwww.acipa-ndl.fr/actualites/ communiques-de-presse/item/650-position-de-l-acipa-sur-la-consultation]. et Vigneux-de-Bretagne, toutes deux riveraines du site prévu pour l'implantation. Au-delà de cet effet de proximité prévisible dans le refus du projet, les géographies du vote «non » livrent d'autres enseignements (figure 3 - planche XI).

\section{Un vote « non " concentré autour de Notre-Dame-des-Landes... et non-négligeable au sud de la Loire}

Sans surprise, la figure 3 (planche XI) montre donc que le niveau du vote «non » est inversement proportionnel à la distance à la commune de Notre-Dame-des-Landes (où $20 \%$ des inscrits ont d'ailleurs voté « oui »). Pour autant, les scores du «non » sont loin d'être négligeables dans l'espace de Nantes-Métropole.

C'est notamment le cas en son centre, à Nantes, où le résultat revêt une importance symbolique toute particulière, à la fois comme capitale régionale et parce que la ville a longtemps été dirigée par l'ancien Premier ministre Jean-Marc Ayrault et qu'elle est aujourd'hui administrée par Johanna Rolland, emblématique du "phénomène de professionnalisation à l'œuvre au PS depuis quelques années, qui se traduit par la promotion d'un nouveau profil d'élus, issus des cabinets des collectivités territoriales ou de la fonction publique locale et formés à la science politique » (Lefebvre, 2014). Au coude-à-coude tout au long de la soirée de dépouillement, c'est finalement le « oui » qui l'emporte de cent voix sur 180000 inscrits. À La Chapelle-sur-Erdre - située au nord de Nantes et dont le maire est le premier secrétaire fédéral du PS en Loire-Atlantique - c'est le «non » qui l'emporte de justesse.

À Rezé, $3^{\text {e }}$ commune de Nantes-Métropole, le « non » l'emporte également à $53 \%$ des suffrages exprimés (soit $25 \%$ des inscrits), alors même que la commune est directement concernée par les nuisances sonores liées à la trajectoire d'approche des avions, avec une pointe à près de $67 \%$ de « non » parmi les bulletins exprimés dans le bureau de vote gentrifié de Trentemoult, pourtant survolé par les avions à seulement 200 mètres d'altitude. Enfin, sur la commune même de Bouguenais où se situe l'aéroport de Nantes-Atlantique - dont l'ancienne maire Françoise Verchère est une figure centrale de l'opposition au projet dans le cadre du Collectif des Élus Doutant de la Pertinence de l'Aéroport (CEDPA) 
- c'est le « oui » qui l'emporte de quelques dizaines de bulletins. Ces villes de 10000 à 30000 habitants ont en commun d'être toutes des bastions de la gauche socialiste, et leurs élus s'y trouvent donc en position de porte-à-faux par rapport aux votes de leurs administrés, votes dont on peut faire l'hypothèse - qui appelle bien entendu confirmation qu'ils expriment autant un désaccord sur les valeurs qui président à un tel projet d'aménagement que des craintes en matière de pérennité de l'emploi si l'aéroport basculait au nord de la Loire.

Si l'on s'éloigne de Nantes-Métropole pour prendre un peu de distance, les corrélations constatées sur l'ensemble du département tendent à confirmer ces premiers constats (tableau 3). Politiquement, le vote «non » est en effet étroitement corrélé aux résultats de la liste EELV aux régionales 2015 (r $=0,81$ ), et très légèrement aux résultats de la liste PS-PRG $(0,18)$ et de la liste PCF-MRC $(0,14)$. Sociologiquement, le vote «non » est associé statistiquement à la présence de populations d'âge moyen $(\mathrm{r}=$ 0,34 avec les 35-49 ans), et surtout à la localisation des populations les mieux dotées du département dans les hiérarchies scolaires $(0,53$ avec les titulaires de Bac+2 et 0,39 avec les diplômés de $2^{\mathrm{e}}$ et $3^{\text {e }}$ cycles universitaires) et socioprofessionnelles $(0,50$ avec les professions intermédiaires et 0,48 avec les cadres et professions intellectuelles supérieures), autant de catégories dont les travaux de sociologie électorale montrent qu'elles constituent aujourd'hui la partie centrale de l'électorat de la gauche socialiste (Lefebvre, Sawicki, 2006), même si l'échelle communale doit inviter à la prudence dans l'interprétation.

\section{Un vote «non » qui souligne les zones de force des gauches dans l'espace intra- urbain}

La cartographie intra-urbaine des résultats du « non », comme les corrélations établies au niveau des bureaux de vote (tableau 3), consolident les conclusions esquissées sur le rapport étroit entre la distribution spatiale du vote «non » et l'implantation des différentes composantes de la gauche. La carte $\mathrm{du}$ « non » rappelle en effet les quartiers nantais qui constituent les zones de force d'EELV lors des scrutins récents (Rivière, 2014a) : une large dorsale nord-sud le long de la ligne de tramway $\mathrm{n}^{\circ} 2$ ainsi

\begin{tabular}{|c|c|c|}
\hline Variables & $\begin{array}{l}\text { Échelle des } \\
\text { communes }\end{array}$ & $\begin{array}{c}\text { Échelle des } \\
\text { bureaux }\end{array}$ \\
\hline $18-24$ ans & NS & 0,26 \\
\hline $25-39$ ans & 0,15 & 0,26 \\
\hline $40-54$ ans & 0,34 & $-0,36$ \\
\hline $55-64$ ans & NS & $-0,36$ \\
\hline $65-79$ ans & $-0,27$ & $-0,18$ \\
\hline 80 ans ou plus & $-0,30$ & NS \\
\hline Sans diplôme & $-0,48$ & $-0,45$ \\
\hline CEP & $-0,47$ & $-0,23$ \\
\hline BEPC & $-0,24$ & $-0,35$ \\
\hline CAP-BEP & NS & $-0,28$ \\
\hline Bac & 0,34 & NS \\
\hline $\mathrm{Bac}+2$ & 0,53 & 0,51 \\
\hline $\mathrm{Bac}+3$ et plus & 0,39 & 0,32 \\
\hline Agriculteurs & $-0,27$ & 1 \\
\hline $\begin{array}{l}\text { Artisans, comm., chefs } \\
\text { entr. }\end{array}$ & 0,15 & NS \\
\hline Cadres, Prof. intel. sup. & 0,48 & 0,34 \\
\hline Prof. Intermédiaires & 0,50 & 0,48 \\
\hline Employés & 0,18 & $-0,28$ \\
\hline Ouvriers & $-0,31$ & $-0,35$ \\
\hline Retraités & $-0,28$ & NS \\
\hline Autres & NS & NS \\
\hline Propriétaires & 0,16 & 0,16 \\
\hline Locataires parc privé & $-0,15$ & 0,44 \\
\hline Locataires parc HLM & & $-0,46$ \\
\hline Logés gratuitement & $-0,16$ & 0,16 \\
\hline Abstention & $-0,16$ & $-0,55$ \\
\hline Blancs & NS & NS \\
\hline Nuls & $-0,35$ & NS \\
\hline $\mathrm{LO}$ & NS & NS \\
\hline $\mathrm{CO}$ & NS & NS \\
\hline PCF-MRC & 0,14 & 0,45 \\
\hline EELV & 0,82 & 0,81 \\
\hline PS-PRG & 0,18 & 0,54 \\
\hline UDB & NS & 0,41 \\
\hline UMP-UDI-MODEM & $-0,20$ & NS \\
\hline UPR & NS & NS \\
\hline DLF & NS & NS \\
\hline FN & $-0,21$ & $-0,30$ \\
\hline
\end{tabular}

Tableau 3 : Coefficients de corrélation entre le vote " non " et des variables sociales et politiques à l'échelle communale et des bureaux de vote nantais

Ecological correlation between the "no » vote and political sociological variables by municipality and by polling station in Nantes 
que les quartiers de Zola, Chantenay ou de l'Île de Nantes. Dans l'espace interne à la capitale régionale, dont la sociologie est spécifique par rapport au reste du département, le vote « non » est ainsi très fortement lié au vote pour la liste $\operatorname{EELV}(\mathrm{r}=0,81)$, ce qui est logique tant cette liste avait mis en avant l'enjeu de l'aéroport dans sa campagne régionale de 2015. Mais le «non » est aussi très présent dans les bureaux de vote qui étaient favorables $(\mathrm{r}=0,54)$ à la liste socialiste menée par Christophe Clergeau en 2015, ainsi qu’à la liste PC-MRC conduite par Alain Paganot $(r=0,45)$. Or, ces deux listes (celle du PS-PRG vis-à-vis de la liste EELV avec laquelle elle fusionnera ${ }^{20}$, et celle du PCF-MRC contre ses partenaires du Front de Gauche) se sont en partie construites sur le soutien au projet d'aéroport.

Dans l'espace nantais, le vote «non » émane donc des quartiers où habite l'électorat des gauches dans leur diversité, le résultat final pouvant indiquer une coupure entre les positions du PS et du PCF et leurs bases électorales, coupure qui devrait constituer un avertissement sérieux dans des villes comme Nantes ou Rezé, laboratoires du socialisme municipal. C'est d'autant plus frappant quand on observe en détail la sociologie des bureaux de vote où le vote « non » est le plus fort. Ses corrélations sont en effet positives avec la présence de populations assez jeunes $(r=0,26$ avec les 18-24 ans et 0,26 avec les 25-39 ans), bien dotées en capital culturel $(0,51$ avec les titulaires d'un $\mathrm{Bac}+2$ et 0,32 avec les $\mathrm{Bac}+3$ et plus), bien insérées dans le monde professionnel $(0,48$ avec les professions intermédiaires et 0,34 avec les cadres et professions intellectuelles supérieures) et plutôt locataires du parc privé $(0,44)^{21}$. Là encore, ce sont des groupes qui renvoient à la clientèle électorale classique de la gauche socialiste urbaine, qu'il s'agisse des villes de l'Ouest dont Nantes est emblématique (Rivière, 2016) ou de l'ensemble des villes du haut de la hiérarchie urbaine nationale (Rivière, 2014b).

20. À noter que le «non » reste fortement corrélé $(\mathrm{r}=0,68)$ avec la liste d'union de la gauche (fusion de la liste PS-PRG et de la liste EELV) présente au second tour du scrutin régional de 2015.

21. Or ces catégories sont précisément celles qui sont les plus surreprésentées dans la sociologie des passagers aériens en France : les tranches d'âges des 15-25 ans (17 \% des passagers) et des 26-35 ans (24\%); les CSP des professions intermédiaires $(25 \%)$ et des cadres et professions intellectuelles supérieures $(24 \%)$. Source : Direction générale de l'Aviation civile, 2010. Enquête Nationale des Passagers Aériens, Ministère de l'Écologie, du Développement durable, des Transports et du logement, Paris, [http://wwww.developpement-durable.gouv.fr/IMG/pdf/ Rapport_Annuel_2010_sans_double_compte_sansrappel2009_pour_internet-1.pdf].

\section{Conclusion - Un SCRUTin QUI NE RÈGLE RIEN?}

Les résultats de cette consultation électorale inédite montrent que le projet de «transfert » de l'aéroport de Nantes est clairement refusé par les habitants du site d'implantation prévu à Notre-Damedes-Landes (commune qui constitue le centre de gravité des votes «non») et pas franchement souhaité par les habitants du site d'implantation actuel de Nantes-Atlantique (dans les communes de Nantes, Rezé ou Bouguenais, les plus concernées par les nuisances de survol, les résultats sont très serrés). Plus largement, cette consultation pose la question de savoir à quelle échelle géographique la légitimité de tels aménagements - désormais qualifiés par le label de GP2I (Grands Projets Inutiles et Imposés) dans le vocabulaire militant - peut être assurée (Subra, 2007).

Bien que ce conflit d'aménagement semble clos du point de vue du pouvoir via le recours au sésame électoral, politiquement la question est toutefois loin d'être réglée. Si on peut faire l'hypothèse que la principale fonction de cette consultation a été de permettre « une forme de réassurance de l'État dans sa prétention à l'exercice et à l'utilisation de son "monopole de la violence physique légitime", pour reprendre la formulation canonique du sociologue Max Weber »(Boudic, 2016), on peut se demander si le gouvernement prendra le risque politique d'une tentative d'évacuation de la ZAD à l'automne 2016. Au moment d'une rentrée parlementaire où l'agenda médiatique sera intégralement tourné vers la pré-campagne du scrutin présidentiel de 2017 (et ses différentes primaires), l'État est-il est prêt à prendre le risque d'une évacuation violente, voire à assumer le coût politique d'éventuels morts à NotreDame-des-Landes, comme ce fut le cas pour Rémi Fraisse, tué sur la ZAD de Sivens? Ou à mobiliser entre 2000 et 2500 policiers, gendarmes, et militaires pendant plusieurs mois non seulement pour évacuer la ZAD mais aussi pour y sécuriser le début de travaux de construction de l'aéroport, et ce dans un contexte de menaces terroristes qui réclament la mobilisation de ces forces de l'ordre ailleurs sur le territoire national? 


\section{Remerciements}

Je tiens à remercier les coordinateurs de ce numéro thématique, Stéphanie Dechézelles (Sciences Po Aix, CHERPA) et Maurice Olive (Aix-Marseille Université, CHERPA) pour avoir accepté cette proposition tardive de note de recherche en lien avec l'actualité récente. Sur le plan méthodologique, l'analyse à l'échelle des bureaux de vote nantais a pu être conduite grâce à une technique développée dans le cadre du programme de recherche CARTELEC (http://www. cartelec.net/), et je tiens à remercier tout particulièrement Sylviano Freire-Diaz (Université de Rouen, IDEES) qui a procédé, dans des délais réduits, à la ventilation des données de l'INSEE dans les bureaux de vote nantais redécoupés en 2015.

\section{Bibliographie}

Barbe F., 2016. La « zone à défendre » de Notre-Dame-desLandes, ou l'habiter comme politique, Norois, n 238-239, p. 109-126.

Beauguitte L., Colange C., 2013. Analyser les comportements électoraux à l'échelle du bureau de vote. Mémoire scientifique de l'ANR CARTELEC, [https://halshs.archivesouvertes.fr/halshs-00839899/document].

Boudic G., 2016. Le référendum au risque de l'instrumentalisation, Place Publique, n ${ }^{\circ}$ 57, p. 28-33.

Braconnier C., Dormagen J.-Y., 2007. La Démocratie de l'abstention. Aux origines de la démobilisation électorale en milieux populaires, Paris, Folio, $460 \mathrm{p}$.

Braconnier C., Mayer N. (dir.), 2015. Les inaudibles. Sociologie politique des précaires, Paris, Presses de Science Po, $250 \mathrm{p}$.

Bussi M., Colange C., Freiré-Diaz S., Jadot A., 2010. Un outil d'analyse électorale en cours de création. CARTELEC, un SIG au niveau des bureaux de vote français, Le monde des cartes. Revue du comité français de cartographie, $\mathrm{n}^{\circ} 205$, p. 81-98.

Charpentier A., Coulmont C., Gombin J., 2014. Un homme, deux voix : le vote par procuration, La Vie des idées, [http:// www.laviedesidees.fr/Un-homme-deux-voix-le-vote-par.html].

Collectif Hérodote, 1976, «Attention : géographie! », Hérodote, $\mathrm{n}^{\circ} 1$, p. 3-7.

Garrigou A., 2006. L'ivresse des sondages, Paris, La Découverte, $128 \mathrm{p}$.
Lefebvre R., 2014. En attendant le «vote sanction »? Les notables socialistes en ordre de bataille, Métropolitiques, [http://www.metropolitiques.eu/En-attendant-le-vote-sanction.html].

Lefebvre R., Sawicki F., 2006. La société des socialistes. Le PS aujourd'hui, Bellecombe-en-Bauges, Éd. du Croquant, $255 \mathrm{p}$.

Lehingue P., 2007. Subunda Coups de sonde dans l'océan des sondages, Bellecombe-en-Bauges, Éd. du Croquant, 271 p.

Lehingue P., 2005. Mais qui a gagné? Les mécanismes de production des verdicts électoraux (le cas des scrutins municipaux), in Lagroye J., Lehingue P., Sawicki F. (dir.), La Mobilisation électorale municipale, Paris, PUF, p. 323-360.

Pailloux A.-L., 2015. Zone d'aménagement différé contre « zone à défendre ». Analyse d'une lutte pour l'autonomie dans/de l'espace rural, Justice spatiale / Spatial justice, $\mathrm{n}^{\circ} 7$, [http://www.jssj.org/article/zone-damenagement-differecontre-zone-a-defendre-analyse-dune-lutte-pour-lautonomiedansde-lespace-rural/].

RAPETTI D., 1985. Vote et société dans la région nantaise. Étude de géographie électorale. 1945-1983, Paris, CNRS Éditions, $212 \mathrm{p}$.

Renard J., Rialland-Juin C., 2013. Le projet d'aéroport de Notre Dame des Landes : les rebonds d'un aménagement conflictuel, Géoconfluences, [http://geoconfluences.ens-lyon. fr/doc/territ/FranceMut/FranceMutDoc 17.htm].

Rialland-Juin C., 2016. Le conflit de « Notre-Dame-desLandes » : les terres agricoles entre réalités agraires et utopies foncières, Norois, no 238-239, p. 133-145.

Rivière J., 2016. Comprendre les configurations électorales internes aux villes de l'Ouest, in Bussi M., Le Digol C., Voillot C. (dir.), Le Tableau politique de la France de l'Ouest d'André Siegfried 100 ans après. Héritages et postérités, Rennes, PUR, p. 183-201.

Rivière J., 2014a. L'abstention, le vote et les inégalités sociales à Nantes, Place publique, no 47, p. 11-17.

Rivière J., 2014b. Les divisions sociales des métropoles françaises et leurs effets électoraux. Une comparaison des scrutins municipaux de 2008, Métropolitiques, [http://www. metropolitiques.eu/Les-divisions-sociales-des.html].

Robinson W.S., 1950. Ecological Correlations and the Behavior of Individuals, American Sociological Review, vol. 15, $\mathrm{n}^{\circ} 3$, p. 351-357, DOI 10.2307/2087176.

Russo L., Beauguitte L., 2014. Aggregation level matters: evidence from french electoral data, Quality \& Quantity, Vol. 48, Issue 2, p. 923-938, doi :10.1007/s11135-012-9814-0.

Subra P., 2007. Géopolitique de l'aménagement du territoire, Paris, A. Colin, 327 p. 

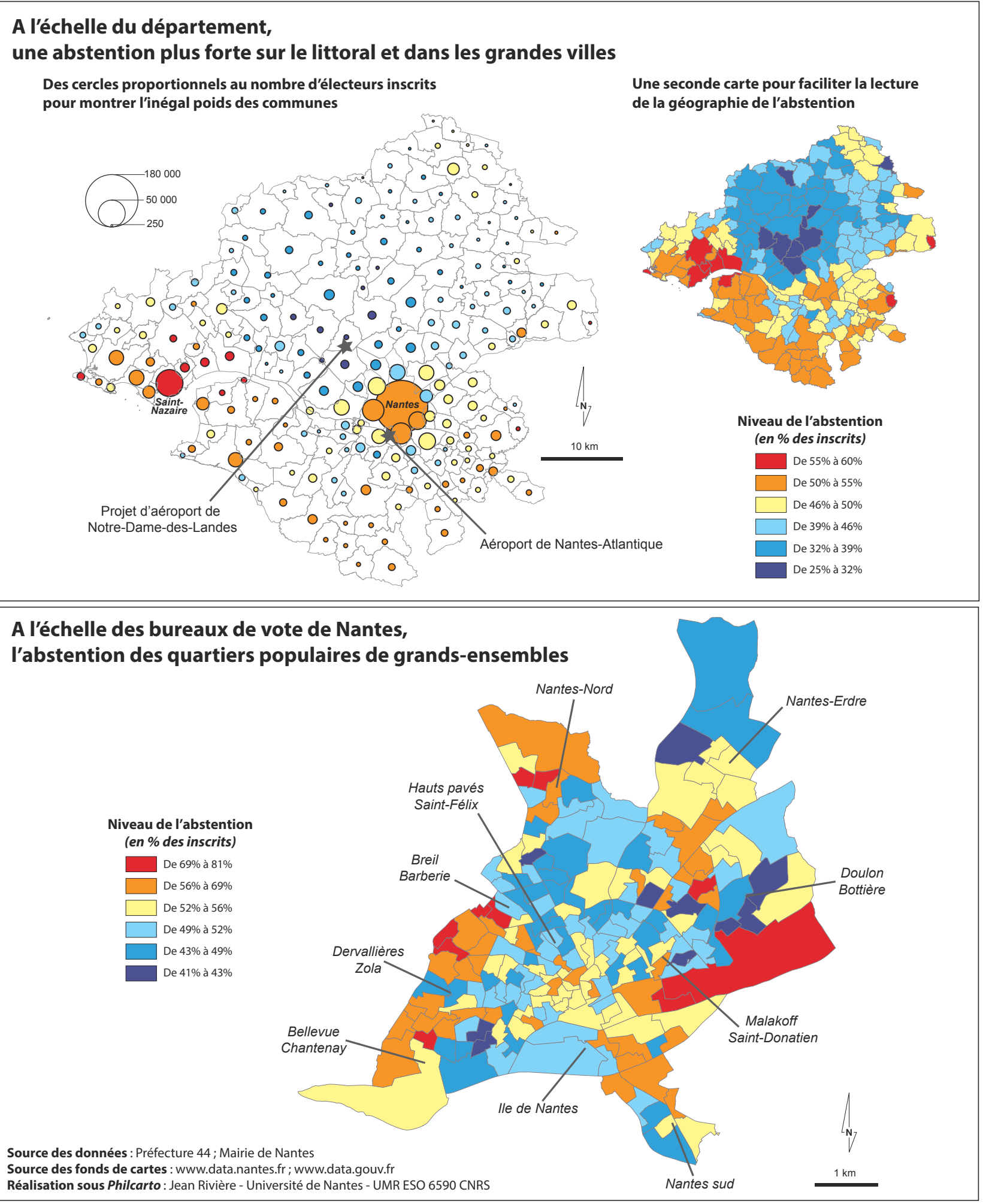

Figure 1 : Géographies de l'abstention Geography of abstention 


\section{A l'échelle du département, un vote " oui » concentré au nord et dans les mondes ruraux}

Des cercles proportionnels au nombre d'électeurs inscrits pour montrer l'inégal poids des communes

pour montrer l'inégal poids des communes
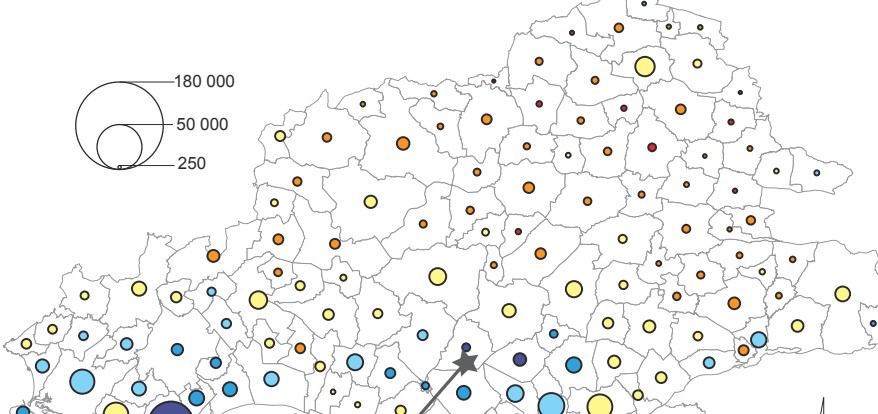

$$
00
$$

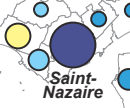

$3_{0}^{0} 0$

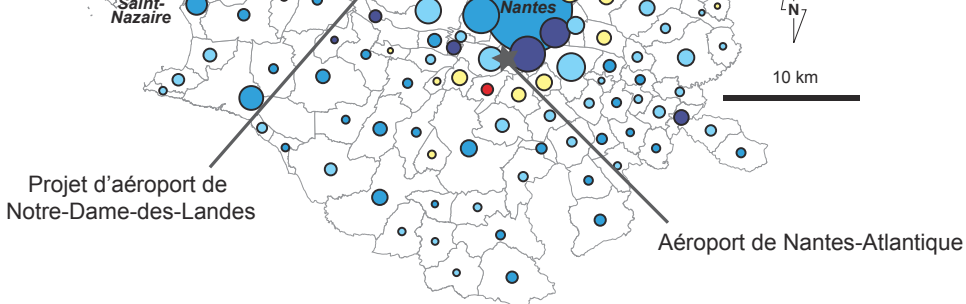

Une seconde carte pour faciliter la lecture de la géographie du vote " oui »

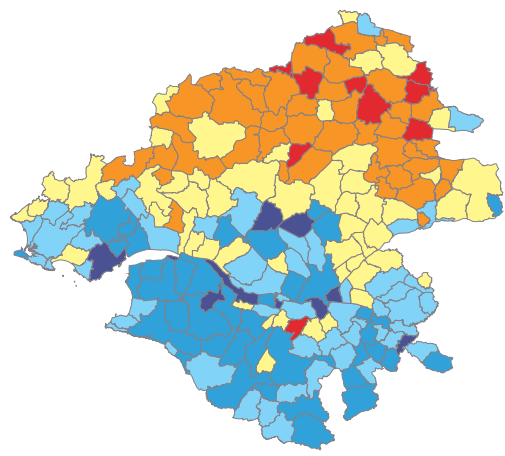

Niveau du vote « oui » (en \% des inscrits)

De $46 \%$ à $52 \%$

De $37 \%$ à $46 \%$

De $31 \%$ à $37 \%$

De $26 \%$ à $31 \%$

De $23 \%$ à $26 \%$

De $18 \%$ à $23 \%$

A l'échelle des bureaux de vote de Nantes, un vote " oui » dans les quartiers favorables aux droites

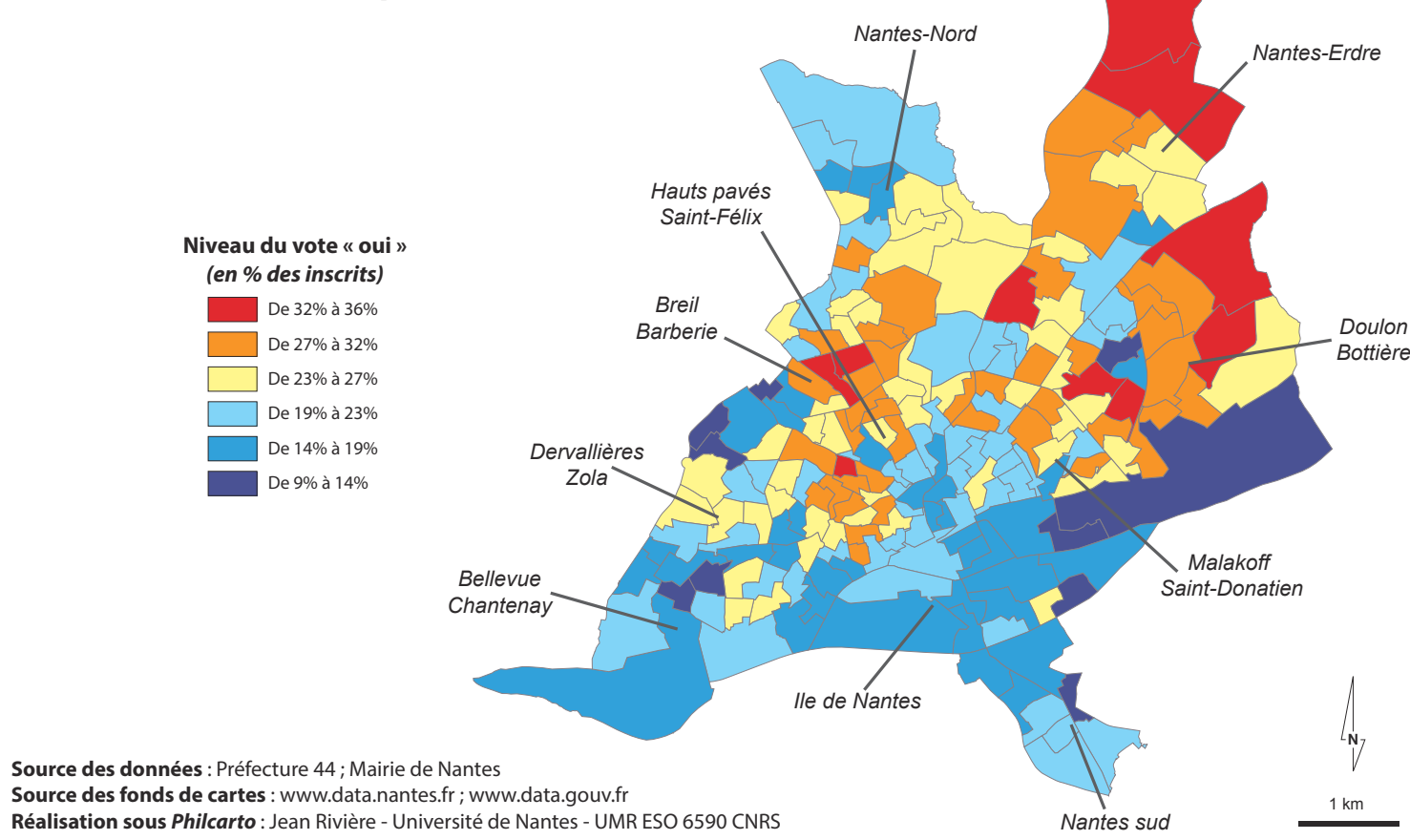

Figure 2 : Géographies du vote « oui » Geography of the "yes" vote 

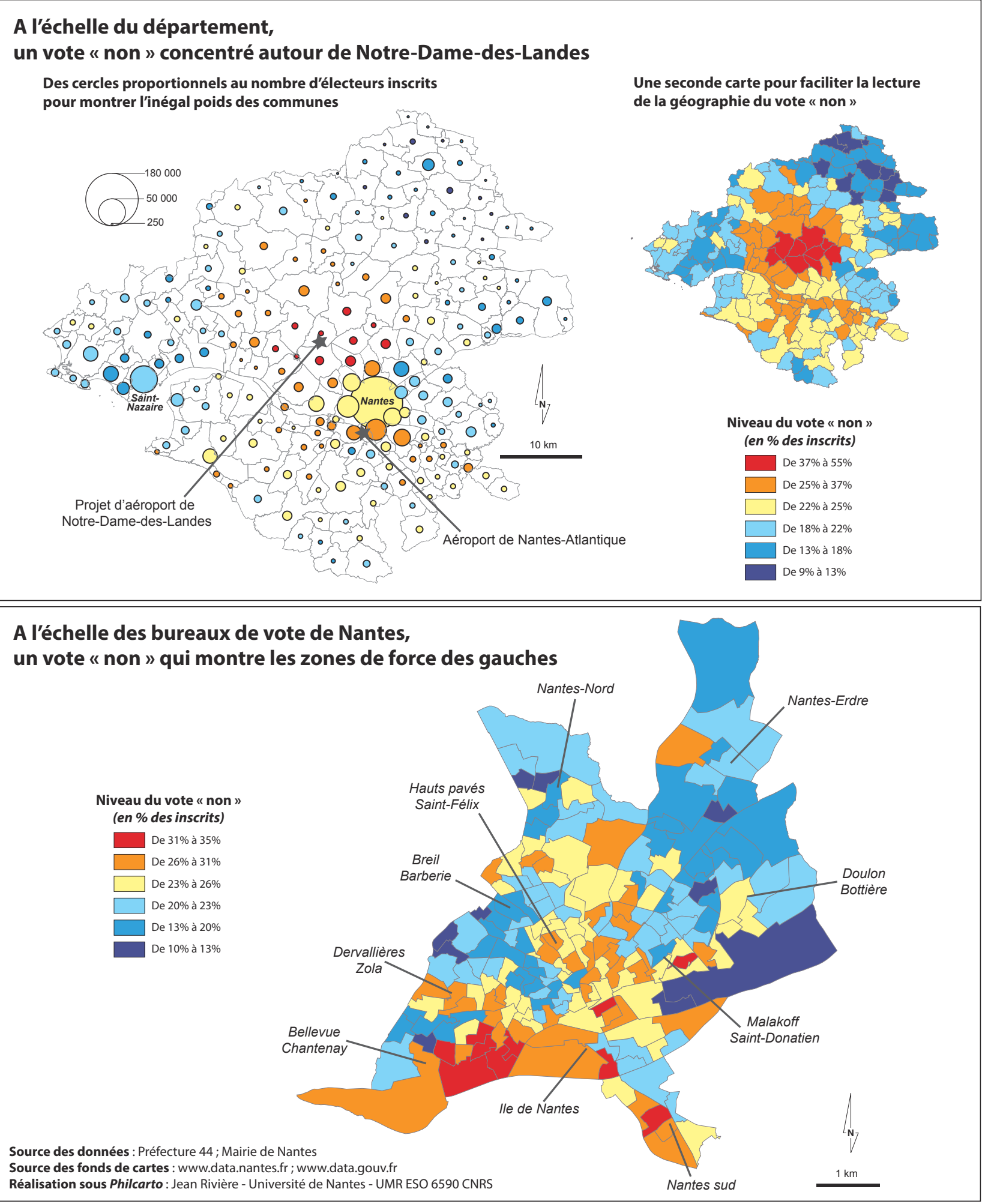

Figure 3 : Géographies du vote «non » Géography of the "no" vote 